\title{
Cardiomyopathies in children: Mitochondrial and Storage disease
}

Dr Gabrielle Norrish ${ }^{1,2}$, Professor Perry M Elliott ${ }^{2,3}$

Centre for Inherited Cardiovascular Diseases, Great Ormond Street Hospital, London UK (1);

Institute of Cardiovascular Sciences University College London, UK (2);

St Bartholomew's Centre for Inherited Cardiovascular Disease, St Bartholomew's Hospital, West Smithfield, London, UK (3)

\section{Corresponding author:}

P. M. Elliott, MBBS; MD; FRCP; FESC; FACC

UCL Institute for Cardiovascular Science

Paul O'Gorman Building room G26

University College London

72 Huntley Street

London, WC1E 6DD

Email: perry.elliott@ucl.ac.uk 


\section{Non-structured abstract}

Inborn errors of metabolism are individually rare but account for up to $10 \%$ of all childhood cardiomyopathies. This group of diseases is extremely heterogeneous in terms of age of onset, presentation and natural history. This review highlights 'red flags' in the presentation, examination or investigations of patients with metabolic storage or mitochondrial disease that can identify particular aetiologies and guide further investigations and management.

\section{Key words}

Cardiomyopathy; Paediatric; Inborn errors of metabolism; Mitochondrial disease 


\section{$\underline{\text { Introduction }}$}

The prevalence of childhood cardiomyopathies in the general population is unknown, but population-based registry studies from North America[1], Australia[2] and Finland[3] report an incidence rate between 0.65 and 12.4 per 100,000. Childhood cardiomyopathies are heterogeneous in terms of morphological phenotype, age of presentation and underlying aetiology. The most common morphological phenotypes are dilated and hypertrophic cardiomyopathy, which constitute approximately $60 \%$ and $25 \%$ of all childhood cases, respectively[4]. Other forms of cardiomyopathy subtypes, including restrictive, are much rarer.

Individually, inborn errors of metabolism (IEM) are rare diseases with an incidence of less than 1 per 100,000 births. However, population studies estimate that their collective incidence approaches 1 in 800 live births [5] and that they may account for up to $10 \%$ of all childhood cardiomyopathies[2, 4]. IEM can be grouped according to the metabolic pathway affected, with specific diseases associated with a characteristic phenotype and natural history [4]. This review focuses on the features and outcomes of childhood cardiomyopathy caused by IEM. It highlights diagnostic clues or 'red flags' and briefly describes the investigations that can identify particular aetiologies and guide further investigations and management.

\section{Presentation}

The presentation of this heterogeneous patient group varies depending on the underlying aetiology and cardiac phenotype. Cardiac involvement may be the first presenting feature (e.g. heart failure symptoms in Pompe disease), be precipitated or exacerbated at times of metabolic decompensation (eg mitochondrial disease), or increasingly diagnosed as part of 
screening for multi-systemic diseases (eg Friedreich ataxia or Mucopolysacharidoses). Patients with HCM secondary to a metabolic disease are more likely to present in infancy (65\%) with symptoms of heart failure at diagnosis[4] compared to those with sarcomeric disease.

The age of presentation is highly variable, but the majority of mitochondrial and storage disorders present during early childhood and cardiac involvement in other IEM is not commonly seen until adolescence (e.g Friedreich Ataxia) or adulthood (e.g. Anderson Fabry). The majority of IEM are multi-system diseases, but isolated cardiac involvement does rarely occur (e.g. Anderson Fabry disease). The presence of dysmorphic features, developmental delay, neurological involvement, myopathy or hepatosplenomegaly should prompt clinicians to consider IEM as a cause of the cardiac disease (table 1).

\section{Cardiac investigations}

Characteristic abnormalities can be seen on the 12-lead resting ECG in patients with storage or mitochondrial diseases. They include ventricular pre-excitation (e.g. Pompe, Danon, PRKAG2, MELAS, MERFF) and extreme QRS voltages (e.g, Pompe) Figure 1. Conduction disease is a common feature of certain storage (e.g. Danon disease) or mitochondrial disease (eg Kearns-Sayre syndrome).

As the cardiac morphological phenotype of IEM is highly variable there are no specific echocardiographic features that are synonymous with a storage or mitochondrial disease. However, individual aetiologies may have characteristic phenotypes such as severe biventricular hypertrophy (Figure 2), concentric hypertrophy Figure 3, DCM and valvular abnormalities Table 1. 


\section{Natural history}

Not surprisingly, given the heterogeneous nature of IEM, the natural history is largely determined by aetiology. However, patients with HCM secondary to IEM have a worse outcome compared to other aetiologies (sarcomeric or malformation syndromes) with 1 and 5-year survival of $53.6 \%$ and $41.7 \%$, respectively[4]. Patients presenting in infancy have a particularly poor prognosis with a 5 -year survival of only $26.3 \%$. Mortality is primarily secondary to heart failure, although certain diseases (e.g. Danon disease) are at risk of malignant arrhythmias and sudden cardiac death[6].

IEMs associated with a dilated phenotype have a better prognosis with survival plateauing over 5 year follow up ( 1 and 5-year survival of 86 and $83 \%$ respectively)[7]. This compares favourably to DCM secondary to neuromuscular disease (5-year survival $57 \%$ ) but is worse than is seen for familial DCM (5-year survival 94\%)[8].

\section{Specific metabolic aetiologies}

The cardiac phenotype of specific metabolic aetiologies associated with childhood cardiomyopathies is described below and summarised in Table 1.

\section{Disorders of glycogen metabolism}

Glycogen storage disorders (GSD) with an estimated incidence of $1 / 20,000-45,000$ live births are caused by abnormalities in the storage, synthesis or breakdown of glycogen. They are the most common type of IEM associated with HCM in childhood and are characterised by multi- 
system involvement (particularly affecting skeletal muscle and the liver) and pre-excitation on a resting ECG (table 1).

Pompe disease (Glycogen Storage Disease Type 2a) is an autosomal recessive disorder caused by deficient lysosomal acid alpha -1,4-glucosidase (GAA) activity which results in intracellular glycogen deposition. It is the most common cause of HCM secondary to an IEM during childhood[4]. An inverse relationship exists between residual enzyme activity and the severity of clinical presentation with infantile, juvenile and late-onset variants recognised. In classical infant-onset disease there is virtual absence or less than 1\% GAA activity in tissues [9] and patients present with progressive multi-systemic disease which includes hypotonia, muscle weakness, hepatomegaly and signs of congestive cardiac failure secondary to severe biventricular hypertrophy. The resting electrocardiogram (ECG) typically shows extremely high QRS voltages and a short PR interval. Historically, patients with infantile Pompe disease had a poor prognosis with early death secondary to cardiorespiratory failure before 1 year of age[9]. However, since 2006, the introduction of enzyme-replacement therapy has extended survival [10]. The mean age of presentation for late onset disease is 28 years, although almost one fifth present under the age of 12 years. Patients with later onset forms have a less severe progressive muscle phenotype, but cardiac involvement is uncommon.

Danon disease is an X-linked storage disorder characterised by a clinical triad of cardiomyopathy, skeletal myopathy and intellectual disability[11]. The disease is caused by mutations in Lysosomal Associated Membrane Protein (LAMP2) which is required for 
chaperone-mediated autophagy[12]. The prevalence of the disease is unknown but LAMP-2 mutations were identified in $1 \%$ of a cohort of predominately adult patients with $\mathrm{HCM}[13]$.

Male patients have an earlier onset of disease, with an average age of 12.2 years compared to $\mathbf{2 7 . 9}$ years for female mutation carriers, and are more severely affected [14]. All male patients have cardiac involvement which is characteristically severe concentric HCM, although approximately $12 \%$ progress to a dilated phenotype. Conduction abnormalities are common ( $86 \%$ of patients) and $69 \%$ of patients have a short PR interval with slurred QRS upstroke on a 12-lead resting ECG. Patients with Danon disease are known to be at high risk for malignant ventricular arrhythmias and sudden cardiac death[6]. Additional extra-cardiac features are common in male patients and include muscle weakness (80-90\%) with an elevated serum creatinine kinase, cognitive impairment (70\%) and retinal involvement comprising peripheral pigmentary retinopathy or pigmentary atrophy $(69 \%)$. In comparison, female mutation carriers have a milder phenotype and are unlikely to present during childhood. A cardiomyopathy is seen in $60-100 \%$ of female mutation carriers with an equal prevalence of dilated (28\%) and hypertrophic (33\%) phenotypes[14]. Twenty seven percent have evidence of ventricular pre-excitation on a 12-lead resting ECG.

Genotype phenotype correlations in Danon disease are incompletely understood, although nonsense or frameshift mutations are reported to have an earlier onset and more severe phenotype compared to splicing or missense mutations[15]. Male patients have a worse prognosis than females with an earlier average age of cardiac transplantation and death (17.9 vs 33.7 years and 19.0 vs 34.6 years, respectively). In a recent series, the oldest male patient 
who did not reach an end-point of transplant or death was 25 years[14]. In women, the disease is progressive with $18 \%$ requiring cardiac transplantation in one series[14].

Mutations in the gamma 2 subunit of the adenosine monophosphate-activated protein kinase (PRKAG2) cause an autosomal dominant disease characterised by ventricular pre-excitation, progressive conduction disease and hypertrophic cardiomyopathy[16]. PRKAG2 mutations result in deposition of a glycogen like substance in transgenic mice[17], but the pathophysiology is poorly understood. The age penetrance of the condition is variable but ECG changes (short PR, ventricular pre-excitation, voltage criteria for LVH) are present in all mutation carriers by 18 years of age[18] (figure 1). Left ventricular hypertrophy develops at a median age of 32 and $22 \%$ of patients have conduction disease by the age of 40 [19]. Biventricular hypertrophy in infancy secondary to heterozygote PRKAG2 mutations has been reported[20]. Mortality in these patients is secondary to or sudden cardiac death or heart failure $[18,19]$.

\section{Lysosomal storage disorders}

Lysosomal storage disorders (LSD) are a heterogeneous group of diseases caused by defects in the metabolism of lipids or glycoproteins leading to the accumulation of substrate within lysosomes. Although individually rare, as a group they occur with an estimated incidence of 1 per 5000-10,000 live births. The LSDs are classified by the type of stored material involved (Lipid storage disorders, mucopolysacchardidoses and mucolipidoses) of which mucopolysaccharidoses (MPSs) most commonly have cardiac involvement. 
MPS are a group of diseases caused by the absence of enzymes responsible for the degradation of glycosaminoglycans (GAG) leading to progressive systemic deposition. The inheritance pattern is variable and includes autosomal dominant, recessive and X-linked forms. In common with other metabolic diseases, MPS have multi-system involvement (table 1). Cardiac disease is reported for all MPS syndromes, but is more common and occurs earlier for MPS I (Hunter), II (Hurler) and VI (Maroteaux-Lamy)[21, 22]. Valvular disease (particularly mitral and aortic valve) is the most frequent cardiac manifestation occurring in $60-90 \%$. The underlying pathophysiology of valve disease is multifactorial and includes GAG accumulation in valve tissue and inflammation. Valvar disease is often progressive with initial valvular insufficiency which evolves to stenosis over time. Other cardiac manifestations include; coronary artery disease[23], which has been described in individuals with all types of MPS; conduction abnormalities (present in $44 \%$ of patients with MPS VI)[23]; concentric HCM (60\%)[22] which may be secondary to systemic hypertension; and LV aneurysms[24]. Rarely, patients with MPS can have a dilated cardiomyopathy phenotype [25]. Enzyme replacement therapy for MPS I, II and VI (laronidase, idursulfase and galsulfase, respectively) has been shown to result in improvement in mobility and airway disease with some studies reporting a decrease in left ventricular hypertrophy[26]. Clinical trials have not shown an improvement in established cardiac valvar disease with ERT[27], but it is hypothesised that earlier treatment may prevent cardiac involvement.

\section{Mitochondrial diseases}


Mitochondrial diseases are clinically and genetically heterogeneous diseases caused by dysfunction of the mitochondrial respiratory chain which is essential for ATP generation. They cause multi-system disease, particularly affecting organs with high energy demands, which includes cardiac muscle.

\section{Inheritance pattern}

The mitochondrial oxidative phosphorylation system comprises 5 multi-subunit enzyme complexes (complexes 1-V) and two electron transporters (coenzyme Q10 and cytochrome C). Although 99\% of these proteins are encoded by nuclear DNA (nDNA), which are inherited in mendelian fashion, $1 \%$ is encoded by mitochondrial DNA (mtDNA), which is maternally inherited. This genetic heterogeneity has important clinical consequences.

Nuclear DNA replicates with each cell division and mutations can be inherited in either autosomal dominant, recessive or X-linked patterns. The clinical phenotype resulting from such mutations is more homogenous as all mitochondria are similarly affected.

In contrast, mtDNA replicates continuously, independently of cell division, and due to an absence of DNA repair mechanisms is at higher risk of somatic mutations. At birth, the mtDNA population of a cell is usually identical (homoplasmic), however during cell division random allocation of daughter mitochondria leads to a mixture of mtDNA (heteroplasmy) with different proportions of wild-type or mutated mtDNA. When a threshold proportion of mutant mtDNA is reached, impaired energy production results in organ dysfunction and clinical manifestations. The exact threshold differs for each tissue and mutation. In this way, the tissue specific level of heteroplasmy has been shown to correlate with clinical phenotype. As a result, the penetrance of mtDNA mutations or rearrangements is highly variable; 
homoplasmic mtDNA mutations will be transmitted to all offspring often with incomplete penetrance, whereas heteroplasmic mtDNA mutations are distributed randomly with clinical effects that are difficult to predict.

Mitochondrial disease can be caused by point mutations or rearrangements (deletions, substitutions, duplications). Approximately $15 \%$ of mitochondrial diseases are cause by a mutation in mtDNA, meaning the majority are caused by mutations in nDNA.

\section{Cardiac disease}

$20-40 \%[28,29]$ of children with mitochondrial diseases have an associated cardiomyopathy. Screening for cardiac involvement is therefore standard care for patients with a confirmed or suspected mitochondrial disease. The majority of patients present under the age of 6 years, but presentation in adulthood is typical for certain mitochondrial diseases [30]. Cardiac manifestations can be precipitated or exacerbated at times of metabolic decompensation such as with intercurrent illness or surgery as this is a time of increased ATP demand. The most common cardiac phenotype associated with mitochondrial disease is concentric HCM ( $60 \%)$, although progression to a dilated phenotype over time is frequently seen. Other phenotypes include a dilated cardiomyopathy ( $30 \%)$ or left ventricular non-compaction with systolic impairment ( 13\%) [29]. Abnormalities on a 12-lead ECG are common and include ventricular pre-excitation (eg MELAS syndrome) and conduction disease (eg Kearns Sayre syndrome).

A summary of the main type of mitochondrial disorders associated with cardiomyopathy is given below and in table 1. 
Mitochondrial syndromes due to mtDNA abnormalities

Mitochondrial syndromes are characteristic constellations of signs and symptoms typically caused by abnormalities in mtDNA. These include Kearns Sayre Syndrome, MELAS (mitochondrial encephalomyopathy, lactic acidosis and stroke like episodes syndrome) , MERRF (myoclonic epilepsy with ragged red fibres syndrome) and Leigh syndrome. In Kearns Sayre syndrome, the principal cardiac manifestation is conduction disease with sudden cardiac death reported in this population[31, 32]. Cardiomyopathy is more common in other mitochondrial syndromes with HCM, DCM and LVNC phenotypes described.

Single or multiple oxidative phosphorylation complex deficiencies Isolated cardiomyopathies and cardiac involvement as part of a multi-systemic disease have been reported in most individual complex deficiencies. Complex I deficiency is the most common single complex deficiency (30\%) associated with childhood onset disease and is typically associated with $\mathrm{HCM}$ and pre-excitation on a resting ECG. Multiple complex deficiencies are associated with heterogeneous disease which can include cardiomyopathies.

\section{Coenzyme Q10 deficiency}

Primary coenzyme Q10 deficiency has a varied clinical presentation which includes encephalomyopathy, myopathy, ataxia and hypertrophic cardiomyopathy. It is important to identify as treatment with Coenzyme Q10 replacement may improve the clinical picture.

Disorders with 3 methylglutaconic aciduria 
Barth syndrome and Senger syndrome are examples of mitochondrial diseases characterised by increased excretion of 3 methylglutaconic aciduria. Barth syndrome is a rare X-linked disease caused by mutations in the TAZ gene characterised by cardiomyopathy, skeletal myopathy, growth retardation and neutropenia[33]. Registry studies report that cardiomyopathy, most frequently dilated or LVNC, occurs in almost all patients (96\%) with 70\% presenting before 1 year of age[34]. Cardiac features may be the presenting manifestation in over two third of patients and can include arrhythmias or sudden cardiac death. Prognosis is poor with a five-year survival of $51 \%$, with death occurring secondary to cardiac failure or infection[35]. As a result, prophylactic antibiotics are an important part of patient management. No specific treatments are currently available, although a phase 2 trial evaluating the safety and efficacy or elamipretide, which has been shown to improve 6minute walk test in primary mitochondrial myopathies[36], is currently underway (TAZPOWER). Cardiac transplantation is controversial, however successful transplantation has been reported[37]. Senger syndrome is an autosomal recessive disease characterised by congenital cataracts, $\mathrm{HCM}$, mitochondrial myopathy and lactic acidosis secondary to mutations in the AGK gene (Figure 2). The neonatal form has a poor prognosis with severe HCM and infantile death secondary to heart failure.

\section{Other disorders of mitochondrial function}

Friedreich ataxia is an autosomal recessive neurodegenerative disease caused by mutations in FXN and is characterised by progressive ataxia, areflexia, sensory neuropathy and HCM. It is the most common spinocerebellar degenerative disease in white Caucasian populations with a prevalence of 1:29,000-1:50,000[38]. Neurological symptoms precede cardiac in the 
majority, although 90\% develop cardiac involvement (concentric hypertrophy) under the age of 18 years[39] (Figure 3). ECG abnormalities are seen in most patients (90\%) which include repolarisation abnormalities, abnormal QRS axis and left ventricular hypertrophy [40] There are conflicting reports regarding the association between disease severity and the length of Frataxin repeat or neurological and cardiac manifestations [41, 42]. The natural history of cardiac involvement is progression to a dilated phenotype with decline in ejection fraction over time[43]. Atrial arrhythmias are seen commonly in patients with advanced disease. The average life expectancy is 29-38 years with death occurring secondary to cardiac causes (heart failure or arrhythmias) in 60\%[44].

\section{Management}

Paediatric patients with mitochondrial disease and cardiomyopathy have an increased mortality compared to those with no cardiac involvement ( $71 \%$ vs $26 \%$ by 40 years)[30]. Mortality is primarily secondary to heart failure, although sudden cardiac death is also reported particularly for certain diseases (eg Kearns Sayre syndrome).

For many mitochondrial diseases, no definitive treatment options are currently available.

Primary coenzyme Q10 deficiency is an exception in whom supplementation can result in dramatic improvement although the response is unpredictable. Vitamins and cofactor supplementation are often used empirically in mitochondrial disease, although a recent cochrane review found little supporting evidence for their efficacy[45][46]. It is recommended that all patients with a mitochondrial disease should be offered receive nutritional supplementation of coenzyme Q10, riboflavin and $\alpha$-lipoic acid[45, 47]. Folinic acid should 
only be considered in those with central nervous involvement and L-carnitine only when there is a documented deficiency.

The mainstay of management is therefore aimed at symptom control with medical management of heart failure symptoms in accordance with published guidelines[48] and implantation of permanent pacing systems for conduction disease[49, 50]. Risk stratification for arrhythmic events should take place routinely and arrhythmias managed with antiarrhythmics or device therapy.[48, 51] The decision to implant a primary prevention implantable cardioverter defibrillator (ICD) should include consideration of the extent of multisystem disease and long-term prognosis. Patients with mitochondrial diseases can successfully undergo cardiac transplantation, however in the presence of multi-systemic disease this can be controversial [37].

\section{Conclusions:}

In conclusion, storage and mitochondrial diseases are an uncommon but important cause of childhood cardiomyopathies. They are extremely heterogeneous in terms of their age of onset, clinical presentation and natural history. However, diagnostic clues can be found in baseline cardiac investigations including a 12-lead resting ECG and echocardiogram. The importance in making a specific diagnosis is two-fold. Firstly, individual diseases have a characteristic natural history which includes heart failure, arrhythmias, conduction disease and SCD. Knowledge of the underlying aetiology can guide clinicians in their management and surveillance of a patient. Additionally, disease specific therapies are increasingly available which can prevent disease progression or even reverse cardiac disease (eg MPS). A structured 
approach to the investigation and management of these patients will result in a greater understanding of the spectrum of cardiac disease. 


\section{$\underline{\text { References }}$}

1. Lipshultz, S.E., et al., The incidence of pediatric cardiomyopathy in two regions of the United States. N Engl J Med, 2003. 348(17): p. 1647-55.

2. Nugent, A.W., et al., The epidemiology of childhood cardiomyopathy in Australia. N Engl J Med, 2003. 348(17): p. 1639-46.

3. Arola, A., et al., Epidemiology of idiopathic cardiomyopathies in children and adolescents. A nationwide study in Finland. Am J Epidemiol, 1997. 146(5): p. 385-93.

4. Colan, S.D., et al., Epidemiology and cause-specific outcome of hypertrophic cardiomyopathy in children: findings from the Pediatric Cardiomyopathy Registry. Circulation, 2007. 115(6): p. 773-81.

5. Sanderson, S., et al., The incidence of inherited metabolic disorders in the West Midlands, UK. Arch Dis Child, 2006. 91(11): p. 896-9.

6. Maron, B.J., et al., Clinical outcome and phenotypic expression in LAMP2 cardiomyopathy. Jama, 2009. 301(12): p. 1253-9.

7. Towbin, J.A., et al., Incidence, causes, and outcomes of dilated cardiomyopathy in children. Jama, 2006. 296(15): p. 1867-76.

8. Pahl, E., et al., Incidence of and risk factors for sudden cardiac death in children with dilated cardiomyopathy: a report from the Pediatric Cardiomyopathy Registry. J Am Coll Cardiol, 2012. 59(6): p. 607-15.

9. Kishnani, P.S., et al., Pompe disease diagnosis and management guideline. Genet Med, 2006. 8(5): p. 267-88.

10. Chen, M., L. Zhang, and S. Quan, Enzyme replacement therapy for infantile-onset Pompe disease. Cochrane Database Syst Rev, 2017. 11: p. Cd011539.

11. Danon, M.J., et al., Lysosomal glycogen storage disease with normal acid maltase. Neurology, 1981. 31(1): p. 51-7.

12. Nishino, I., et al., Primary LAMP-2 deficiency causes $X$-linked vacuolar cardiomyopathy and myopathy (Danon disease). Nature, 2000. 406(6798): p. 906-10.

13. Charron, P., et al., Danon's disease as a cause of hypertrophic cardiomyopathy: a systematic survey. Heart, 2004. 90(8): p. 842-6.

14. Boucek, D., J. Jirikowic, and M. Taylor, Natural history of Danon disease. Genet Med, 2011. 13(6): p. 563-8.

15. D'Souza R, S., et al., Danon disease: clinical features, evaluation, and management. Circ Heart Fail, 2014. 7(5): p. 843-9. 
16. Gollob, M.H., et al., Identification of a gene responsible for familial Wolff-ParkinsonWhite syndrome. N Engl J Med, 2001. 344(24): p. 1823-31.

17. Arad, M., et al., Transgenic mice overexpressing mutant PRKAG2 define the cause of Wolff-Parkinson-White syndrome in glycogen storage cardiomyopathy. Circulation, 2003. 107(22): p. 2850-6.

18. Murphy, R.T., et al., Adenosine monophosphate-activated protein kinase disease mimicks hypertrophic cardiomyopathy and Wolff-Parkinson-White syndrome: natural history. J Am Coll Cardiol, 2005. 45(6): p. 922-30.

19. Thevenon, J., et al., High prevalence of arrhythmic and myocardial complications in patients with cardiac glycogenosis due to PRKAG2 mutations. Europace, 2017. 19(4): p. 651-659.

20. Kelly, B.P., et al., Severe hypertrophic cardiomyopathy in an infant with a novel PRKAG2 gene mutation: potential differences between infantile and adult onset presentation. Pediatr Cardiol, 2009. 30(8): p. 1176-9.

21. Fesslova, V., et al., The natural course and the impact of therapies of cardiac involvement in the mucopolysaccharidoses. Cardiol Young, 2009. 19(2): p. 170-8.

22. Lin, S.M., et al., Cardiovascular abnormalities in Taiwanese patients with mucopolysaccharidosis. Mol Genet Metab, 2014. 111(4): p. 493-8.

23. Braunlin, E.A., et al., Cardiac disease in patients with mucopolysaccharidosis: presentation, diagnosis and management. J Inherit Metab Dis, 2011. 34(6): p. 118397.

24. Oudit, G.Y., et al., Left ventricular aneurysm in a patient with mucopolysaccharidosis type VI (Maroteaux-Lamy syndrome): clinical and pathological correlation. Cardiovasc Pathol, 2007. 16(4): p. 237-40.

25. Wiseman, D.H., et al., Management of mucopolysaccharidosis type IH (Hurler's syndrome) presenting in infancy with severe dilated cardiomyopathy: a single institution's experience. J Inherit Metab Dis, 2013. 36(2): p. 263-70.

26. Muenzer, J., Early initiation of enzyme replacement therapy for the mucopolysaccharidoses. Mol Genet Metab, 2014. 111(2): p. 63-72.

27. Lin, H.Y., et al., Cardiac structure and function and effects of enzyme replacement therapy in patients with mucopolysaccharidoses I, II, IVA and VI. Mol Genet Metab, 2016. 117(4): p. 431-7.

28. Holmgren, D., et al., Cardiomyopathy in children with mitochondrial disease; clinical course and cardiological findings. Eur Heart J, 2003. 24(3): p. 280-8.

29. Scaglia, F., et al., Clinical spectrum, morbidity, and mortality in 113 pediatric patients with mitochondrial disease. Pediatrics, 2004. 114(4): p. 925-31.

30. Holmgren, D., Cardiomyopathy in children with mitochondrial disease Clinical course and cardiological findings. European Heart Journal, 2003. 24(3): p. 280-288.

31. Channer, K.S., et al., Cardiomyopathy in the Kearns-Sayre syndrome. Br Heart J, 1988. 59(4): p. 486-90.

32. Khambatta, S., et al., Kearns-Sayre syndrome: a case series of 35 adults and children. Int J Gen Med, 2014. 7: p. 325-32.

33. Jefferies, J.L., Barth syndrome. Am J Med Genet C Semin Med Genet, 2013. 163c(3): p. 198-205. 
34. Roberts, A.E., et al., The Barth Syndrome Registry: distinguishing disease characteristics and growth data from a longitudinal study. Am J Med Genet A, 2012. 158a(11): p. 2726-32.

35. Rigaud, C., et al., Natural history of Barth syndrome: a national cohort study of 22 patients. Orphanet J Rare Dis, 2013. 8: p. 70.

36. Karaa, A., et al., Randomized dose-escalation trial of elamipretide in adults with primary mitochondrial myopathy. Neurology, 2018. 90(14): p. e1212-e1221.

37. Mangat, J., et al., Successful cardiac transplantation in Barth syndrome--single-centre experience of four patients. Pediatr Transplant, 2007. 11(3): p. 327-31.

38. Bourke, T. and D. Keane, Friedreich's Ataxia: a review from a cardiology perspective. Ir J Med Sci, 2011. 180(4): p. 799-805.

39. Child, J.S., et al., Cardiac involvement in Friedreich's ataxia: a clinical study of 75 patients. J Am Coll Cardiol, 1986. 7(6): p. 1370-8.

40. Schadt, K.A., et al., Cross-sectional analysis of electrocardiograms in a large heterogeneous cohort of Friedreich ataxia subjects. J Child Neurol, 2012. 27(9): p. 1187-92.

41. Weidemann, F., et al., The cardiomyopathy in Friedreich's ataxia - New biomarker for staging cardiac involvement. Int J Cardiol, 2015. 194: p. 50-7.

42. Dutka, D.P., et al., Marked variation in the cardiomyopathy associated with Friedreich's ataxia. Heart, 1999. 81(2): p. 141-7.

43. Kipps, A., et al., The longitudinal course of cardiomyopathy in Friedreich's ataxia during childhood. Pediatr Cardiol, 2009. 30(3): p. 306-10.

44. Tsou, A.Y., et al., Mortality in Friedreich ataxia. J Neurol Sci, 2011. 307(1-2): p. 46-9.

45. Pfeffer, G., et al., Treatment for mitochondrial disorders. Cochrane Database Syst Rev, 2012(4): p. Cd004426.

46. Kearney, M., et al., Pharmacological treatments for Friedreich ataxia. Cochrane Database Syst Rev, 2016(8): p. CD007791.

47. Parikh, S., et al., Diagnosis and management of mitochondrial disease: a consensus statement from the Mitochondrial Medicine Society. Genet Med, 2015. 17(9): p. 689701.

48. Kirk, R., et al., The International Society for Heart and Lung Transplantation Guidelines for the management of pediatric heart failure: Executive summary. [Corrected]. J Heart Lung Transplant, 2014. 33(9): p. 888-909.

49. Epstein, A.E., et al., 2012 ACCF/AHA/HRS focused update incorporated into the ACCF/AHA/HRS 2008 guidelines for device-based therapy of cardiac rhythm abnormalities: a report of the American College of Cardiology Foundation/American Heart Association Task Force on Practice Guidelines and the Heart Rhythm Society. J Am Coll Cardiol, 2013. 61(3): p. e6-75.

50. Brignole, M., et al., 2013 ESC Guidelines on cardiac pacing and cardiac resynchronization therapy. Rev Esp Cardiol (Engl Ed), 2014. 67(1): p. 58.

51. Elliott, P.M., et al., 2014 ESC Guidelines on diagnosis and management of hypertrophic cardiomyopathy: the Task Force for the Diagnosis and Management of Hypertrophic Cardiomyopathy of the European Society of Cardiology (ESC). Eur Heart J, 2014. 35(39): p. 2733-79. 
